\title{
Patient satisfaction with the laborist model of care in a large urban hospital
}

\author{
This article was published in the following Dove Press journal: \\ Patient Preference and Adherence \\ 15 March 2013 \\ Number of times this article has been viewed
}

\author{
Sindhu K Srinivas' \\ Anna $O$ Jesus 1 \\ Elene Turzo' \\ Dominic A Marchiano² \\ Harish M Sehdev² \\ Jack Ludmir ${ }^{2}$ \\ 'Maternal and Child Health Research \\ Program, Department of Obstetrics \\ and Gynecology, Perelman School of \\ Medicine, University of Pennsylvania, \\ Philadelphia, PA, USA; ${ }^{2}$ Pennsylvania \\ Hospital, University of Pennsylvania \\ Perelman School of Medicine. \\ Philadelphia, PA, USA
}

Background: The obstetric practice environment is evolving to include more laborists staffing obstetric units, with the hope of improving quality of care and provider satisfaction, yet there are scant data on the impact of a laborist care model on patient satisfaction or delivery outcomes. We sought to assess patient satisfaction after implementation of the laborist model of obstetric care in a large urban teaching hospital.

Methods: Postpartum patients were asked to complete an anonymous survey assessing their satisfaction with care, particularly with regard to the laborist model. Survey questions included rating the overall experience of labor and delivery. All responses were based on a five-point Likert scale. Press-Ganey results were compared from before and after initiation of the model. Descriptive statistics were used to analyze the results.

Results: Post-laborist implementation obstetric and delivery experience surveys were collected from 4166 patients, representing a 54\% response rate. Ninety percent of patients reported that they were highly satisfied with the overall experience in the labor and delivery unit. A subgroup was asked to rate their experience with the practitioner for their current delivery. Of the 687 respondents, $75 \%$ answered excellent, 18\% answered good/very good, and 3.4\% answered neutral. Eighty-five percent of this subgroup stated that they were informed during prenatal care that they may be delivered by someone other than the practitioner or group that they saw during the pregnancy. Thirty-seven percent $(n=1553)$ of the total respondents reported that they had had a previous delivery at this institution, $97 \%(n=1506)$ of whom stated "yes" to having their next delivery at this institution. Press-Ganey results were similarly favorable in both time periods (91.3 $[\mathrm{n}=811]$ versus $93.4[\mathrm{n}=747], P=0.08)$.

Conclusion: Patient satisfaction does not appear to be adversely affected by initiation of the laborist model. Additional research is needed to understand further the implications of this model for provider satisfaction, and maternal and neonatal outcomes.

Keywords: labor, delivery, laborist model, patient satisfaction

\section{Introduction}

Over four million women give birth in the US annually, with the vast majority of deliveries taking place in the hospital setting. Historically, obstetrics was practiced by a solo practitioner who was on-call 24 hours a day for his/her patients. This obstetrician would see patients in the office, and would then be interrupted to perform a delivery for another patient on the labor and delivery unit. Because of a variety of practice changes, technologic advances, and financial pressures, the solo practitioner is becoming obsolete, particularly in urban areas.

The concept of the hospitalist, a term coined in 1996 by Robert Wachter, refers to a physician whose primary focus is the care of hospitalized patients. ${ }^{1}$ In part due to
Correspondence: Sindhu K Srinivas Maternal and Child Health Research Program, Department of Obstetrics and Gynecology, University of Pennsylvania Perelman School of Medicine, 42I Curie Boulevard, BRB II/III I353,

Philadelphia, PA 19104, USA

$\mathrm{Tel}+$ I 2158980825

Fax + I 215 5735408

Email ssrinivas@obgyn.upenn.edu 
market pressures, the number of hospitalists providing inpatient medical care for both children and adults has increased over the last 15 years, with currently over 20,000 practicing hospitalists. Following the success of the hospitalist concept, the laborist concept was introduced nearly a decade ago as a plausible model of obstetric care delivery. Physicians are employed by hospitals to staff labor and delivery, with the underlying premise being continuous coverage for labor and delivery without competing clinical duties. However, much controversy exists over the attributes of this model. The laborist model represents a significant change in the way obstetrics has been experienced and practiced from both a patient and provider perspective. Therefore, this model of care delivery has been adopted by only a small number of labor and delivery units, although its use appears to be growing.

The variety of potential positive and negative attributes of the laborist model is without direct evidence, and largely based on theoretic plausibility and extrapolations from the hospitalist experience. ${ }^{2}$ Possible positive attributes include: 24-hour coverage of labor and delivery with heightened surveillance; dedicated coverage without the distraction of other clinical duties; improved team work; improved ability to respond to emergencies; decreased sleep deprivation leading to better outcomes; reduction in liability claims; improved work hours; and improved family-work life balance. Potential negative aspects that are worrisome to the profession of obstetrics include: lack of continuity of care; disagreement between inpatient and outpatient care providers regarding management; decreased patient satisfaction, leading to loss of patients; reimbursement issues and reduced pay; worse outcomes, especially in high-risk patients, due to lack of knowledge of the patient from antenatal care and increased hand-offs; and over-medicalization of the natural birth process by excessive vigilance. ${ }^{3}$

As reported in several recent news articles, ${ }^{4,5}$ the concern regarding reduced patient satisfaction has been at the forefront of the potential negative attributes of the laborist model. Although central to all medical encounters, the doctor-patient relationship is especially poignant in the birth experience from the point of view of the woman and her family. As a result, the perception that the laborist model could lead to a lack of familiarity with the provider, who will share in the joy of his/her respective patients' deliveries, could reduce patient satisfaction. However, if this model provides safer and improved quality of care, then patient satisfaction could improve despite the purposeful discontinuity introduced by the laborist model.
Two recent studies demonstrate the current state of laborists in the delivery of obstetric care. In a survey of National Perinatal Information Center/Quality Analytic Services member hospitals, nearly $40 \%$ of the 74 member hospitals employ laborists. ${ }^{6}$ In addition, $25 \%$ of respondents to a 2010 American College of Obstetricians and Gynecologists membership survey identified as either laborists or hospitalists. ${ }^{7}$ As evidenced by these studies, the obstetric practice environment is evolving to include more laborists staffing obstetric units, yet there are scant data on the impact of a laborist care model on patient satisfaction or delivery outcomes.

Pennsylvania Hospital, a university-owned teaching hospital with nearly 5000 deliveries per year, is one example of the laborist model in use. The obstetric population at Pennsylvania Hospital is comprised of women from Philadelphia and the surrounding suburbs, and includes maternal transports from the referring area. The hospital underwent a restructuring of provider coverage on labor and delivery on July 1, 2008, in order to provide a more streamlined approach and presence on the labor and delivery unit, with the stated goals of providing safer obstetric care and potentially reducing liability. Prior to the laborist model, six obstetric practices delivered at Pennsylvania Hospital: three private physician practices; a midwifery practice; a residency clinic practice; and Health Center patients who present to Pennsylvania Hospital for delivery. One attending from each of the three private practices was on-call for a given 24-hour shift, but he/she might have also had office hours or gynecologic surgeries scheduled simultaneously. The residents were supervised by one of the three on-call private attendings while managing patients. A midwife was also on-call for his/her own practice.

Prior to implementation of the laborist system, a one-year multidisciplinary working group that included members of the different covering obstetric groups, midwives, nursing personnel, and administration met to discuss the details of this new model of care. The findings were presented to patient focus groups for feedback and included the stated rationale for the implementation of this new model of care delivery. The bylaws of the Department of Obstetrics and Gynecology were changed, stating that attending physicians could not provide hospital care after a 24-hour coverage shift. In addition, it was made mandatory that the responsible attending obstetrician or midwife had to be present on the labor and delivery unit once a woman was admitted in active labor. During the first year of implementation of the laborist model, four laborist physicians were hired to rotate 24-hour shifts during which they are responsible for patients on the labor floor and in the antepartum unit. In this current 
model, participating private physicians' call schedules were combined into a single pool so that a single private physician is assigned to the labor floor for a 24-hour shift in addition to the laborist physician. Similar to the laborist provider, this private physician is solely responsible for laboring patients without competing outpatient duties. In addition, one of the 10 midwives is also scheduled to cover the labor floor (12-hour shifts). This scheduled overlap of responsibilities enables three committed licensed providers to share the volume of laboring patients, and resident education and supervision, with the hope of improving provider satisfaction and quality of care as well as reducing liability. The primary aim of this study was to assess patient satisfaction after initiation of the laborist model at this large urban institution.

\section{Materials and methods}

On admission to Pennsylvania Hospital, each patient is assigned to one of three providers (laborist, private physician, midwife), with attention towards fair distribution of workload, appropriate clinical level, and patient preferences. Under the supervision of the two physicians, all non-midwife labor and delivery patients are managed by a group of 4-5 residents: the intern cares for postpartum patients and attends routine deliveries; the second-year manages the labor floor and attends routine deliveries; the third-year manages the triage unit and attends complicated deliveries; and the fourth-year supervises the other residents while managing resident clinic practice patients as the "acting attending". Prior to the laborist model, the on-call physicians were assigned in a rotating manner to cover resident patients, while caring for their own practice's patients as well. Residents are now supervised evenly by either the private on-call physician or the laborist.

After the introduction of laborists in July 2008, postpartum patients were asked to complete a survey assessing their satisfaction with care, particularly with regards to the laborist model. Surveys were distributed by nurses on the postpartum floor between September 2008 and April 2010, and were collected by the postpartum unit secretary without any patient identifiers prior to patient discharge. In the years during which the surveys were distributed, the number of deliveries per fiscal year (FY) at Pennsylvania Hospital were 4688 in FY 2009 and 4638 in FY 2010. All postpartum patients who delivered at Pennsylvania Hospital between September 2008 and April 2010 were eligible for participation. Institutional review board approval was obtained from Pennsylvania Hospital.

Survey questions included rating of the overall experience on the labor and delivery suite and providing information regarding age and race. In additional, patients were queried about whether they had experienced a prior delivery at Pennsylvania Hospital and, if so, they were asked to rate the labor and delivery experience based on their prior delivery and if they would choose to deliver at Pennsylvania Hospital again. All responses to questions requiring patients to rate their labor and delivery experience were based on a five-point Likert scale of poor, fair, good, very good, and excellent. The data from the surveys was entered and organized in a database for analysis. Responses were collapsed into poor/fair and good/very good based on distribution of responses for improved interpretation of results. Response percentages were rounded to two digits.

Further, in order to compare patient satisfaction before and after implementation of laborists, Press-Ganey results from before (January 1, 2006 to December 31, 2007) and after (January 1, 2009 to December 31, 2010) laborist model initiation were compared. The Press-Ganey report is a nationally utilized patient satisfaction survey distributed to patients following discharge from hospital. Women were queried anonymously and immediately after their hospitalization to minimize recall bias with increasing time between administration and survey completion. Survey results directly related to labor and delivery in both time periods were compared, and included information about patient impression of the courtesy of the childbirth unit, labor and delivery staff introduction of themselves, nursing assistance, promptness and support during labor, and how staff answered questions and concerns after cesarean sections. The overall labor and delivery score is the average of every survey respondent's overall labor and delivery score, which is the average of the respondent's score for all the questions she answered relating to labor and delivery. Press-Ganey converts the respondent's 1-5-point score to a 0-100-point score.

The delivery statistics for FYs 2007-2010 were obtained from Pennsylvania Hospital for the purpose of comparing the patient population composition before and after implementation of the laborist model. Information regarding patient age, race, insurance, and marital status was provided.

Descriptive statistics were performed. The $t$-test was used to compare the Press-Ganey report results for overall rating of labor and delivery before and after implementation of the laborist model. All statistical analyses were performed using Stata version X.0 (College Station, TX, USA).

\section{Results}

A comparison of the demographics between the populations for those who delivered before initiation of the laborist model, those who delivered after initiation, and the survey 
respondents is described in Table 1. The mean subject age was 28 years across the time periods. The racial distribution remained similar, with a slight decrease in the white and black populations and a small increase in the Asian and Hispanic populations. There was a decrease in Blue Cross coverage and a rise in Medicaid and Medicare patients. There was also a $2 \%$ decrease in patients who identified as married. Of the 2564 patients who responded to education level, 14\% had less than a high school diploma, 21\% were high school graduates, $19 \%$ had some college education, and $44 \%$ had a college degree or graduate degree.

Post-laborist implementation obstetric and delivery experience surveys were collected from 4166 patients, representing a 54\% response rate. Overall, $90 \%$ of the 4166 women surveyed reported that they were highly satisfied with the overall experience in the labor and delivery unit. Specifically, $60 \%(n=2500)$ rated their experience as excellent, $30 \%(\mathrm{n}=1250)$ as $\operatorname{good} /$ very good, $1.8 \%(\mathrm{n}=75)$ as neutral, $2.5 \%(n=104)$ poor/fair, and $5.7 \%(n=237)$ did not respond. Eighty-one percent of the respondents reported that they received prenatal care from a physician at Pennsylvania Hospital, $5.5 \%$ at a city health center, $8.1 \%$ other, $5.3 \%$ did not answer, and $0.1 \%$ received no prenatal care.
From the total sample of 4166 patients, $37 \%(n=1553)$ reported that they had had a previous delivery at this institution. When asked "Who delivered your baby?" during the previous delivery, 49\% $(n=759)$ answered "Physician or midwife who provided prenatal care who you had met during pregnancy (in your practice)," 3.8\% $(n=60)$ answered "Physician or midwife who you had not met during your pregnancy," $31 \%(\mathrm{n}=483)$ answered "Resident physician or nurse," 6.3\% $(\mathrm{n}=98)$ answered "Question not applicable" or did not answer the question, $6.2 \%(\mathrm{n}=92)$ answered "Other", and $3.7 \%(n=57)$ answered that "their baby was delivered by more than one provider".

When these 1553 women were asked to rate their current overall labor and delivery experience, $60 \%(n=928)$ rated their experience as excellent, $31 \%(n=479)$ as good/very good, $1.9 \%(n=31)$ as neutral, $2.8 \%(n=44)$ as poor/fair, and $4.3 \%(n=71)$ did not answer the question or responded N/A. When this subgroup was asked "Would you consider delivering at this hospital again?", 97\% $(n=1506)$ replied "yes" to having their next delivery at the institution, $1.6 \%(n=25)$ "maybe," and $1.4 \%(\mathrm{n}=22)$ "no".

Given that one of the big concerns with the laborist model is the lack of a relationship with the delivering

Table I Demographic information before and after initiation of the laborist model

\begin{tabular}{|c|c|c|c|}
\hline & $\begin{array}{l}\text { Pre-laborist } \\
n=10,106\end{array}$ & $\begin{array}{l}\text { Post-laborist } \\
n=9326\end{array}$ & $\begin{array}{l}\text { Survey } \\
\text { respondents* }\end{array}$ \\
\hline Mean age (years) & 28.6 & 28.5 & 28.8 \\
\hline \multicolumn{4}{|l|}{ Race } \\
\hline American Indian/Alaskan native & $0.12 \%(\mathrm{II})$ & $0.26 \%(24)$ & $0.34 \%(9)$ \\
\hline Asian & $5.7 \%(580)$ & $6.6 \%(614)$ & $7.0 \%(179)$ \\
\hline Black/African American & $39 \%(3906)$ & $38 \%(3503)$ & $37 \%(949)$ \\
\hline Hispanic & $9.0 \%(909)$ & $11 \%(1072)$ & - \\
\hline Other & $2.2 \%(219)$ & $0.53 \%(49)$ & $\mathrm{I} .6 \%(4 \mathrm{I})$ \\
\hline Unknown & $0.39 \%(39)$ & $1.3 \%(124)$ & II\% (282) \\
\hline White & $44 \%(4442)$ & $42 \%(3940)$ & $43 \%(1102)$ \\
\hline \multicolumn{4}{|l|}{ Insurance } \\
\hline Managed medicaid & $36 \%(3622)$ & $36 \%(3396)$ & - \\
\hline Managed care & $36 \%(3589)$ & $35 \%(3309)$ & - \\
\hline Blue cross & $18 \%(\mid 824)$ & $16 \%(1,483)$ & - \\
\hline Medicaid or pending & $5.1 \%(5 I I)$ & $6.1 \%(569)$ & - \\
\hline Penn employee & $3.4 \%(343)$ & $3.7 \%(346)$ & - \\
\hline Other & $1.2 \%(152)$ & $1.5 \%(15 \mathrm{I})$ & - \\
\hline Medicare & $0.64 \%(65)$ & $0.88 \%(82)$ & - \\
\hline \multicolumn{4}{|l|}{ Marital status } \\
\hline Divorced & $0.45 \%(45)$ & $0.29 \%(27)$ & - \\
\hline Married & $51 \%(5 \mid 14)$ & $49 \%(4582)$ & - \\
\hline Separated & $0.32 \%(32)$ & $0.23 \%(21)$ & - \\
\hline Single & $49 \%(49 \mid 2)$ & $50 \%(469 I)$ & - \\
\hline Unknown & $0.010 \%(1)$ & $0.0 \%(0)$ & - \\
\hline Widowed & $0.020 \%(2)$ & $0.054 \%(5)$ & - \\
\hline
\end{tabular}

Notes: $*_{n}=4166$ (3479 provided age, 2562 provided race), all other demographics presented were not canvassed in the survey and are based on data collected in hospital. 
provider, a random subgroup of patients was asked, "How would you rate the experience with the practitioner for your current delivery?" Of the 687 respondents, $75 \%$ $(\mathrm{n}=515)$ answered excellent, $18 \%(\mathrm{n}=124)$ answered good/very good, $3.4 \%(\mathrm{n}=23)$ answered neutral, $1.3 \%$ $(\mathrm{n}=9)$ answered poor/fair, and 2.3\% $(\mathrm{n}=16)$ did not answer the question.

The same subgroup of 687 women was asked, "Were you informed during your prenatal care that you may be delivered by someone other than the practitioner (or his/her partner) whom you saw during your pregnancy?" Eighty-five percent $(n=583)$ responded "yes". When asked "Did you expect to be delivered by a certain practitioner?", 61\% $(n=420)$ of respondents answered "no".

Next, in order to compare the pre-laborist and postlaborist time periods more directly, Press-Ganey results were compared. Overall rating of labor and delivery was similarly favorable in both time periods $(91.3[\mathrm{n}=811]$ pre-laborist versus $93.4[\mathrm{n}=747]$ post-laborist, $P=0.08)$.

\section{Discussion}

Implementation of the laborist model creates a large shift in the way providers and patients experience obstetrics. Since its inception, there have been positive and negative attributes reported for this model. One significant negative relates to patient satisfaction, given the purposeful discontinuity between outpatient and inpatient care in the traditional laborist model.

To the authors' knowledge, this study is the first to evaluate patient satisfaction after implementation of this model in order to address this potential negative attribute. We evaluated a large number of patients who answered a survey about the newly implemented laborist model. We found that over $90 \%$ of women rated their experience as good/very good or excellent, and the overwhelming majority who experienced a delivery in both time periods rated their experience similarly.

Despite its strengths and the reassurance it provides regarding patient satisfaction with the laborist model, this study is not without limitations. First, it was a survey of postpartum women after implementation, so lacks a prelaborist model comparison. However, Press-Ganey scores were similar in both time periods. These findings are not attributable to any other additional systematic changes that were made during the time period examined. Further, we had a subgroup of more than 1500 women who experienced both the pre and post time periods. Their results mirrored the results of the overall sample.
Second, as with any survey study, it is possible that the respondents represent a biased group, making the responses less generalizable. This seems unlikely, given the reasonable response rate of $54 \%$ and the overall large sample size of 4166 . Women with negative experiences may have chosen not to respond for fear of being penalized while in the hospital, or they may have minimized their feelings toward the experience by rating their overall experience as neutral. Further, those most concerned about this model may have chosen not to deliver at this hospital and would be missed by the survey entirely. However, demographics before and after implementation of the laborist model were generally similar with regards to racial, financial class, and marital status distribution.

Third, this survey was performed at a single urban teaching hospital, with a specific method for utilizing laborists, and so cannot be fully generalized to all hospitals utilizing laborists. However, this model represents one form of laborist implementation with reassuring satisfaction results, suggesting other models of implementation, including hybrid models, are likely to have similar satisfaction results.

Lastly, the surveys do not provide information about what type of provider delivered care to the women who were satisfied or dissatisfied with their experience, so any correlation between the subset of providers and level of satisfaction cannot be deduced. In the last decade in the US, there has been a significant increase in home births along with an increased prevalence of cesarean deliveries in hospitals and a patient perception of over-medicalization of the birthing process. ${ }^{8}$ Notably, there has been an increase in the proportion of US women who have had a previous cesarean delivery who have then chosen home births for later deliveries, suggesting poor satisfaction with the hospital delivery experience. ${ }^{9}$ Perpetual conflict exists between the goals of patient satisfaction and patient safety, with some experts in bioethics claiming the medicalization of the birthing process to be limiting to patient autonomy..$^{10}$ Therefore, it is of utmost importance to understand the impact of the laborist model on patient satisfaction. One hope is that use of the laborist might provide increased safety and allow for increased focus on individual patient care, marrying the goals of provider and patient.

This study's greatest strength is its unique glimpse of patient satisfaction with the laborist model. Since the introduction of the laborist concept nearly a decade ago, there has been no formal assessment of patient satisfaction. As the use of the laborist model continues to rise, patient satisfaction and perception of the model must be taken into consideration. Our findings suggest that concerns regarding 
decreased patient satisfaction may not be as great an issue as initially considered. This survey demonstrates a high level of patient satisfaction.

Patient expectations might be a factor contributing to the observed patient satisfaction. At our institution, the planned patient focus groups prior to implementation of the laborist model, with clear education and explanation of the model, may have played a role in the high patient satisfaction observed in our study. In addition, a subgroup analysis shows that the vast majority of women were made aware that their practitioner might not deliver them, and this information seems to have affected their expectations, because the majority also did not expect to be delivered by a certain practitioner. If a woman is adequately informed about the nature of the laborist model, she may feel prepared for the possibility of being delivered by someone who was not her provider during her prenatal care, thereby minimizing the disappointment that could arise if that were to be the case. The high level of patient satisfaction after implementation of the laborist model might be reflective of the level of patient understanding regarding their expectations of the childbirth experience.

While our findings are positive, additional research is needed. Future work should focus on continued assessment of patient satisfaction in different practice settings and with different variants of the laborist model implemented. Research is also needed examining maternal and neonatal outcomes, liability impact, provider satisfaction, and graduate medical education as a result of this model. This rigorous and comprehensive approach to evaluation is required to weigh adequately the positives and negatives of implementing this model. The laborist model represents a major evolution in the practice of obstetrics from the provider perspective and in how birth is experienced from a patient and family perspective. With additional evaluation, we can create a vision for maternal and child health that will enable us to develop and promote health services and systems designed to improve the quality of maternal and child care, and to increase patient satisfaction with the birthing experience.

\section{Acknowledgment}

This research was supported by a grant from the Maternal and Child Health Bureau (R40MC17164, to SKS as principal investigator).

\section{Disclosure}

The authors report no conflicts of interest in this work.

\section{References}

1. Wachter RM, Goldman L. The emerging role of "hospitalists" in the American health care system. N Engl J Med. 1996;335(7):514-517.

2. Srinivas SK, Lorch SA. The laborist model of obstetric care: we need more evidence. Am J Obstet Gynecol. 2012;207(1):30-35.

3. Yates J. It's safety first in our laborist program, lifestyle improvement second or third. $O B G$ Management. 2008;20(12):15-16.

4. Silverman RE. "Freebirthing" and "laborists": new birthing options. Wall Street Journal. April 7, 2009. Available from: http://blogs.wsj.com/ juggle/2009/04/07/freebirthing-and-laborists-new-birthing-options/. Accessed February 18, 2013.

5. Lewis S. Specialist hospitalists: could they be the answer to the challenge of call panels? ED Manag. 2008;20:6.

6. Srinivas SK, Shocksnider J, Caldwell D, Lorch S. Laborist model of care: who is using it? J Matern Fetal Neonatal Med. 2012;25(3):257-260.

7. Funk C, Anderson BL, Schulkin J, Weinstein L. Survey of obstetric and gynecologic hospitalists and laborists. Am J Obstet Gynecol. 2010;203:177. e1-e4.

8. MacDorman MF, Menacker F, Declercq E. Trends and characteristics of home and other out-of-hospital births in the United States, 1990-2006. Natl Vital Stat Rep. 2010;58(11):1-14.

9. MacDorman MF, Menacker F, Declercq E. Trends and characteristics of home vaginal birth after cesarian delivery in the United States and selected states. Obstet Gynecol. 2012;119(4):737-744.

10. Bergeron V.The ethics of cesarean section on maternal request: a feminist critique of the American College of Obstetricians and Gynecologists' position on patient-choice surgery. Bioethics. 2007;21(9):478-487.
Patient Preference and Adherence

\section{Publish your work in this journal}

Patient Preference and Adherence is an international, peer-reviewed, open access journal focusing on the growing importance of patient preference and adherence throughout the therapeutic continuum. Patient satisfaction, acceptability, quality of life, compliance, persistence and their role in developing new therapeutic modalities and compounds to

\section{Dovepress}

optimize clinical outcomes for existing disease states are major areas of interest. This journal has been accepted for indexing on PubMed Central. The manuscript management system is completely online and includes a very quick and fair peer-review system. Visit http://www.dovepress.com/ testimonials.php to read real quotes from published authors. 